\title{
Visualizing and profiling lipids in the OVLT of fat-1 and wild type mouse brains during LPS-induced systemic inflammation using AP-SMALDI MSI
}

Janne Bredehöft ${ }^{1,+}$, Dhaka Ram Bhandari ${ }^{2,+}$, Fabian Johannes Pflieger ${ }^{1,+}$, Sabine Schulz ${ }^{2}$, Jing X. Kang ${ }^{3}$, Sophie Layé ${ }^{4}$, Joachim Roth ${ }^{1 \S}$, Rüdiger Gerstberger ${ }^{1}$, Konstantin Mayer ${ }^{5}$, Bernhard Spengler ${ }^{2}$, and Christoph Rummel ${ }^{1 *} \S$

${ }^{1}$ Institute of Veterinary Physiology and Biochemistry, Justus Liebig University Giessen, Frankfurter Strasse 100, D-35392, Giessen, Germany

${ }^{2}$ Institute of Inorganic and Analytical Chemistry, Justus Liebig University Giessen, Heinrich-Buff-Ring 17, D-35392, Giessen, Germany

${ }^{3}$ Laboratory for Lipid Medicine and Technology, Department of Medicine, Massachusetts General Hospital and Harvard Medical School, 149-13th Street, Charlestown, MA 02129, USA

${ }^{4}$ UMR 1286, NutriNeuro: Laboratoire Nutrition et Neurobiologie Intégrée, Institut National de la Recherche Agronomique, Université de Bordeaux, Bordeaux, France ${ }^{5}$ University of Giessen and Marburg Lung Center (UGMLC), Justus Liebig University Giessen, Klinikstrasse 33, Giessen, D - 35392, Germany

${ }^{*}$ Corresponding author: Christoph.D.Rummel@vetmed.uni-giessen.de

${ }^{\S}$ Affiliation: Center for Mind, Brain and Behavior (CMBB), University of Marburg and Justus Liebig University Giessen, Germany ${ }^{+}$these authors contributed equally to this work 
Supporting Information

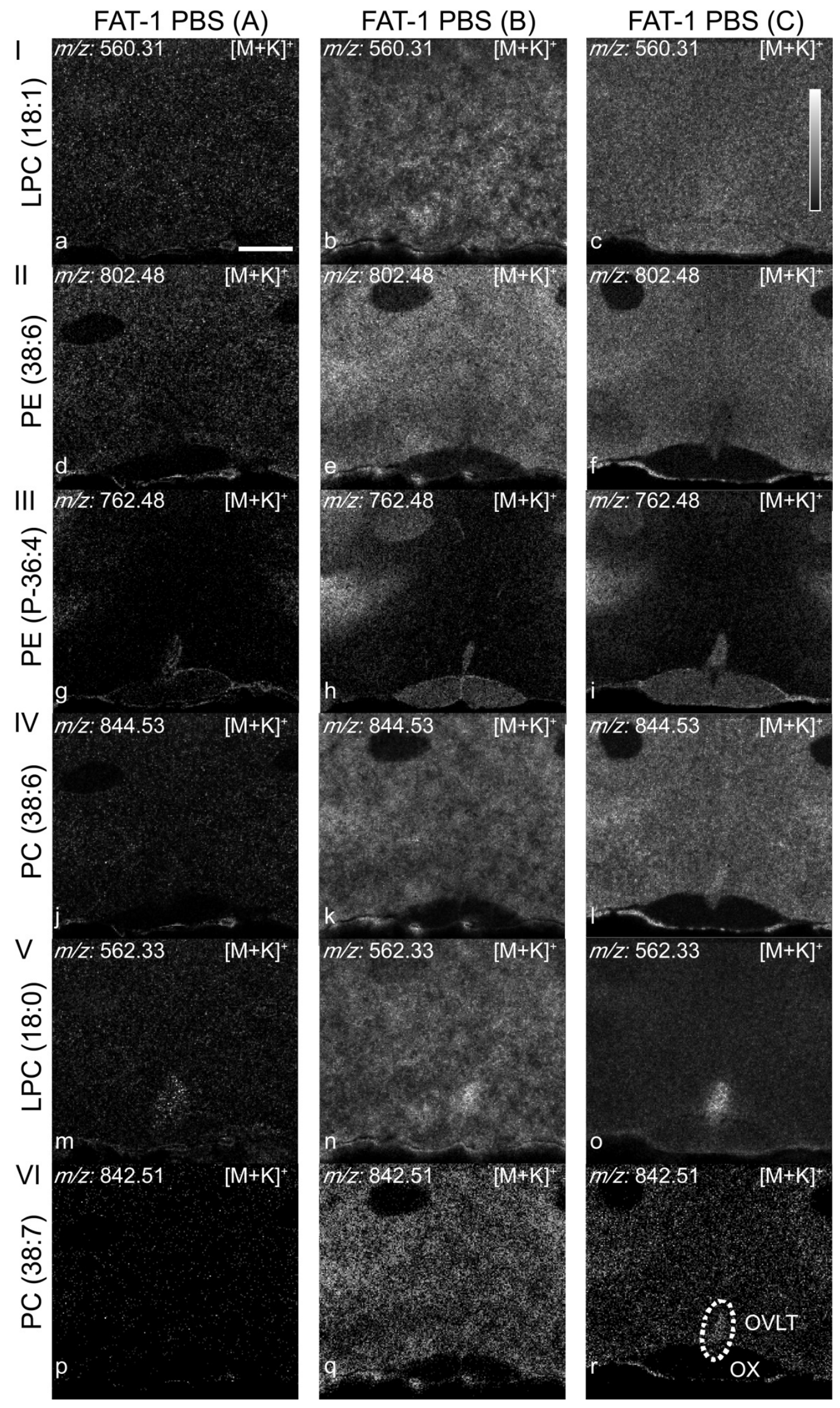




\section{Supplementary Figure 1}

Biological replicates of three PBS treated fat-1 mice (A-C) at the level of the vascular organ of the lamina terminalis (OVLT; white dotted ellipse in (r)) show highly conserved distribution patterns for the lipids displayed.

(I) LPC (18:1) is homogeneously distributed in the three biological replicates ((a)-(c)). (II) The signal for PE (38:6) is reduced in the OVLT of all three animals ((d)-(f)) whereas strong accumulation in the region of interest ((g)-(i)) can be seen for (III) PE (P-36:4). (IV) PC (38:6) showed slightly increased signal intensity in the OVLT ((j)-(I)). The distribution of (V) LPC (18:0) was characterized by a defined signal (white) in the region of the OVLT relative to surrounding tissue of all three measurements $((\mathrm{m})-(\mathrm{o}))$. (VI) PC (38:7) was equally distributed in two out of three replicates $((p)-(r))$.

Ion images exhibit potassium adducts of lipids at $10 \mu \mathrm{m}$ step size and $200 \times 200$ pixels per image. All images were measured in positive-ion mode. Images in this case are not presented in an inverted scale as done for the Figures in the paper to visualization raw data for comparison $($ white $=$ high signal intensity; black, = low signal intensity, see also intensity scale between $0-100 \%$ in (c)). Mass accuracy was better than $2 \mathrm{ppm}$ (RMSE), and an image bin width of $m / z= \pm 5 \mathrm{ppm}$ was used for image generation. Scale bar in (a) represents $0.5 \mathrm{~mm}$ and applies to all images. OX $=$ optic nerve $(r)$. 


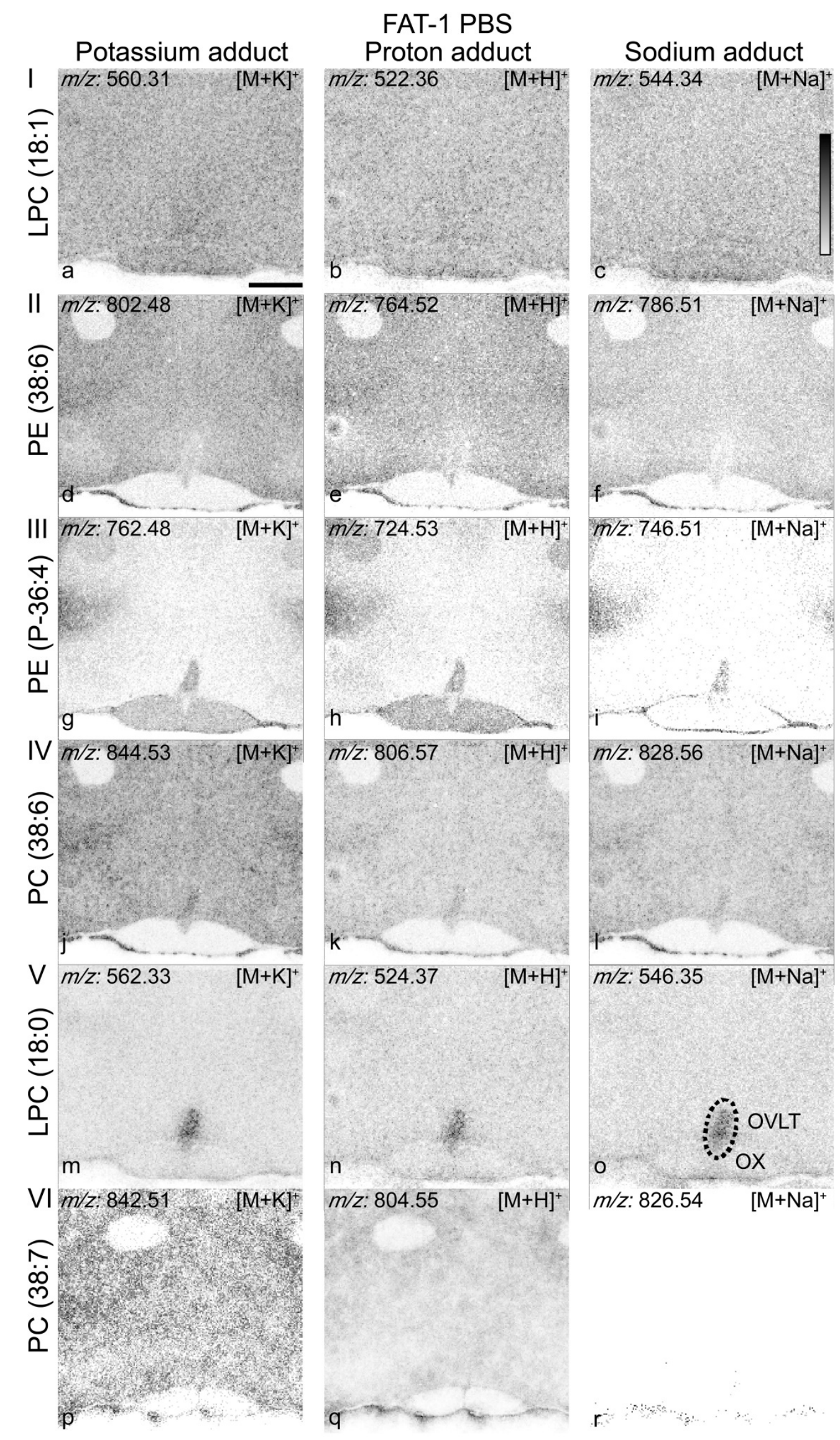

Supplementary Figure 2 
To demonstrate the accuracy of our analyses and to document our workflow, we display examples for adducts of some of the most important lipids described in the manuscript and shown in Figure 1, namely, LPC (18:1), PE (38:6), PE (P-36:4), PC (38:6), LPC (18:0), PC (38:7). The images displayed clearly confirm that the signal peaks of different adducts of one lipid show the same distributional patterns and even intensities, for at least two of three adducts. (I) The signals for LPC (18:1) are homogeneously distributed ((a)-(c)). (II) PE (38:6) adducts are decreased in the vascular organ of the lamina terminalis (OVLT; black dotted ellipse in (o)) and the preoptic region $((\mathrm{d})-(\mathrm{f}))$. Clear high signals in the OVLT are present for (III) PE (P36:4) and (V) LPC (18:0) adducts, (g)-(i) and (m)-(o). (IV) Slightly increased signals in the OVLT can be depicted for adducts of PC (38:6) ((j)-(l)) whereas (VI) PC (38:7) was equally distributed in the OVLT and preoptic area in two out of three adducts $((p)-(q))$ and showed low abundance in the sodium adduct $((r))$.

Ion images exhibit potassium adducts in column one, hydrogen adducts in the second column and sodium adducts in the third column from left to right. All images show $10 \mu \mathrm{m}$ step size and $200 \times 200$ pixels and were measured in positive-ion mode. Images are presented in an inverted scale for better visualization (black = high signal intensity, white = low signal intensity, see also intensity scale between $0-100 \%$ in (c)). Mass accuracy was better than $2 \mathrm{ppm}$ (RMSE), and an image bin width of $m / z= \pm 5$ ppm was used for image generation. Scale bar in (a) represents $0.5 \mathrm{~mm}$ and applies to all images. $\mathrm{OX}=$ optic nerve $(0)$. 


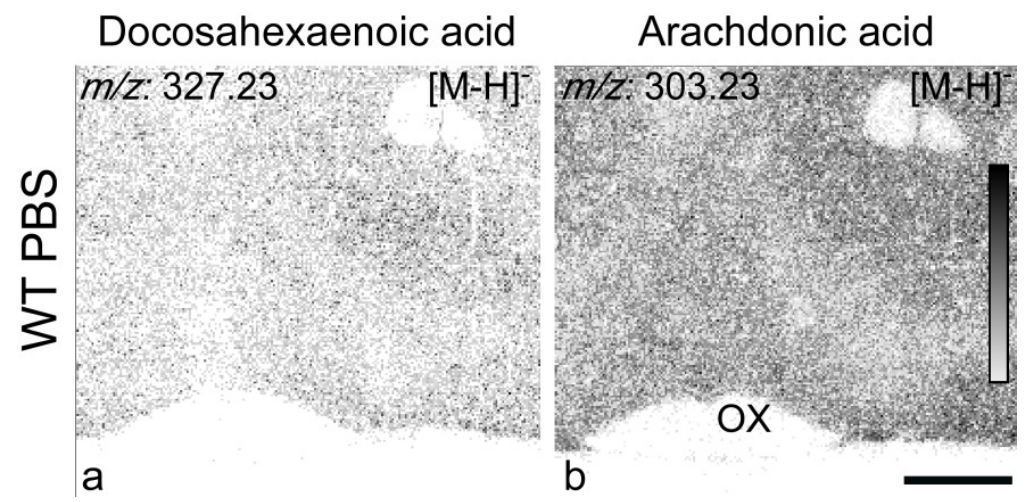

\section{$\underline{\text { Supplementary Figure } 3}$}

Homogeneous distribution pattern of docosahexaenoic acids (DHA) and arachidonic acid (AA) in the area investigated while nerve fibers (optic nerve or anterior commissure) showed less signals. Ion images exhibit mass - hydrogen adducts. All images show $10 \mu \mathrm{m}$ step size and $215 \times 200$ pixels and were measured in negativeion mode. Images are presented in an inverted scale for better visualization (black = high signal intensity, white = low signal intensity, see also intensity scale between 0 $100 \%$ in (b)). Mass accuracy was better than $10 \mathrm{ppm}$ (RMSE), and an image bin width of $m / z= \pm 10 \mathrm{ppm}$ was used for image generation. Scale bar in (b) represents $0.5 \mathrm{~mm}$ and applies to all images. $\mathrm{OX}=$ optic nerve (b). 
a)

MSMS publication \#31-40 RT: 0.65-0.84 AV: 10 NL: 7.37E

80 - Choline

Choline

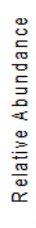

T: FTMS + p NSI Full ms2 560.30@hod20.00 [100.00-600.00]

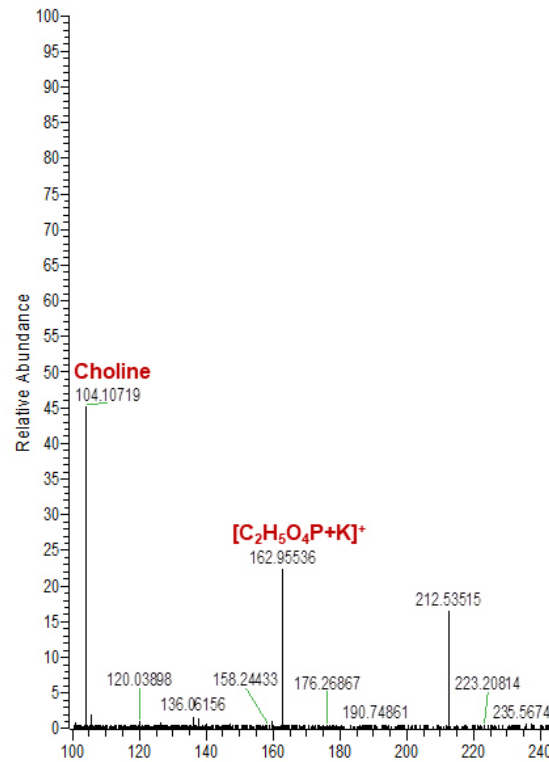

212.71699
$[\mathrm{LPC}(16: 0)+\mathrm{K}]^{+}$

NL 59

475.22077

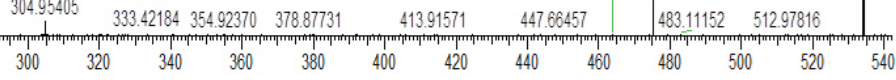

$\mathrm{m} / \mathrm{z}$

$[\operatorname{LPC}(18: 1)+K]^{+}$
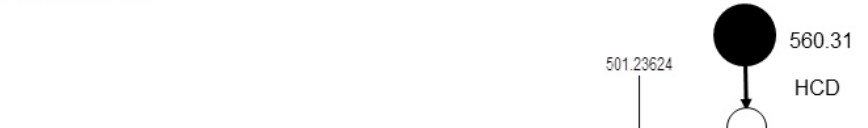

NL 59

$[\mathrm{M}+\mathrm{K}]^{+}$

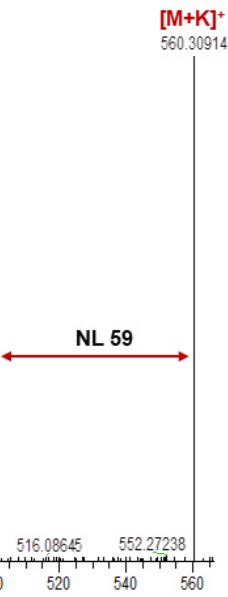
423.98043 \begin{tabular}{l|l}
406.07702 & 445.75345
\end{tabular}

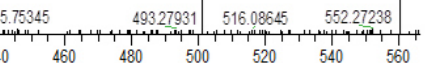


c)

MSMS publication \#80-94 RT: 1.73-2.02 AV: 15 NL: 1.70 E 3

T: FTMS + p NSI Full ms2 562.30@hod20.00 [100.00-600.00]

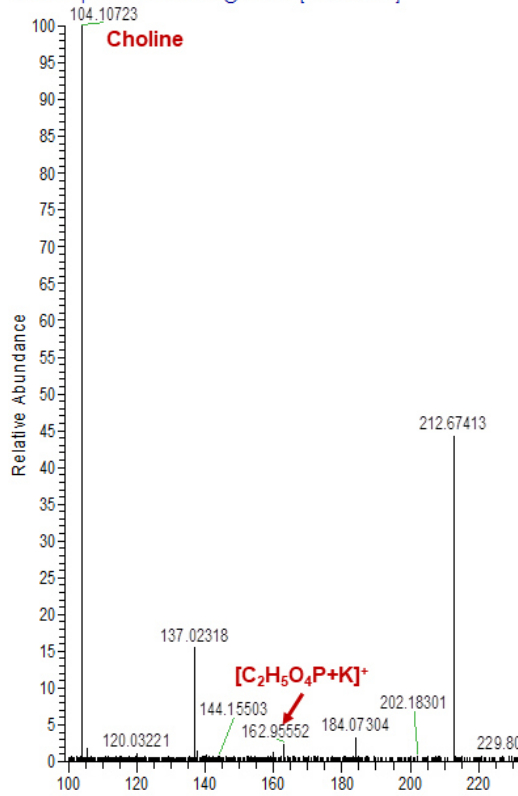

d)

\section{$[\mathrm{PC}(36: 4)+\mathrm{K}]^{+}$}

MSMS publication \#181-228 RT: 4.00-4.36 AV: 18 NL: 5.11E4

T: FTMS + p NSI Full ms2 820.50@hcd20.00 [100.00-1000.00]

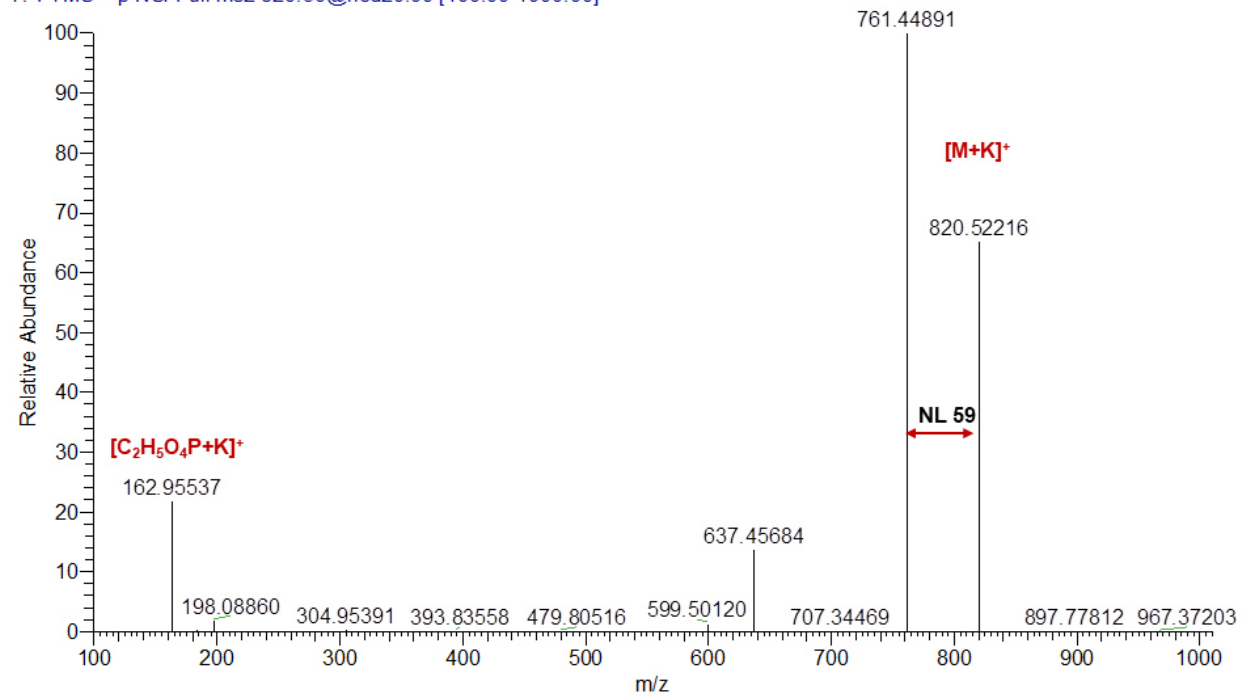

562.33

$\mathrm{HCD}$ 
e)

\section{$[\mathrm{PC}(38: 6)+\mathrm{K}]^{+}$}

MSMS publication \#156 RT: 3.38 AV: 1 NL: $2.80 E 4$

T: FTMS + p NSl Full ms2 844.60@hcd20.00 [99.60-1000.00]

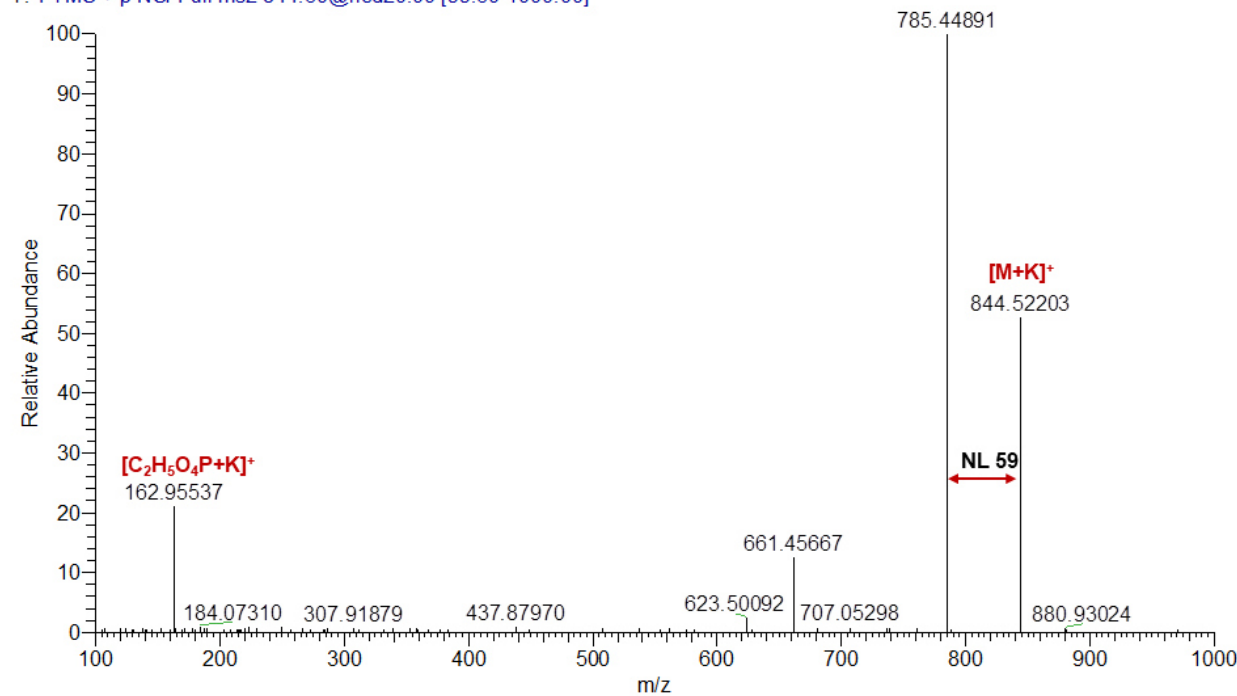

\section{$[\mathrm{PC}(38: 4)+\mathrm{K}]^{+}$}

MSMS publication \#262-281 RT: 5.68-6.13 AV: 20 NL: 6.11E4

T: FTMS + p NSI Full ms2 848.50@hcd10.00 [100.00-1000.00]

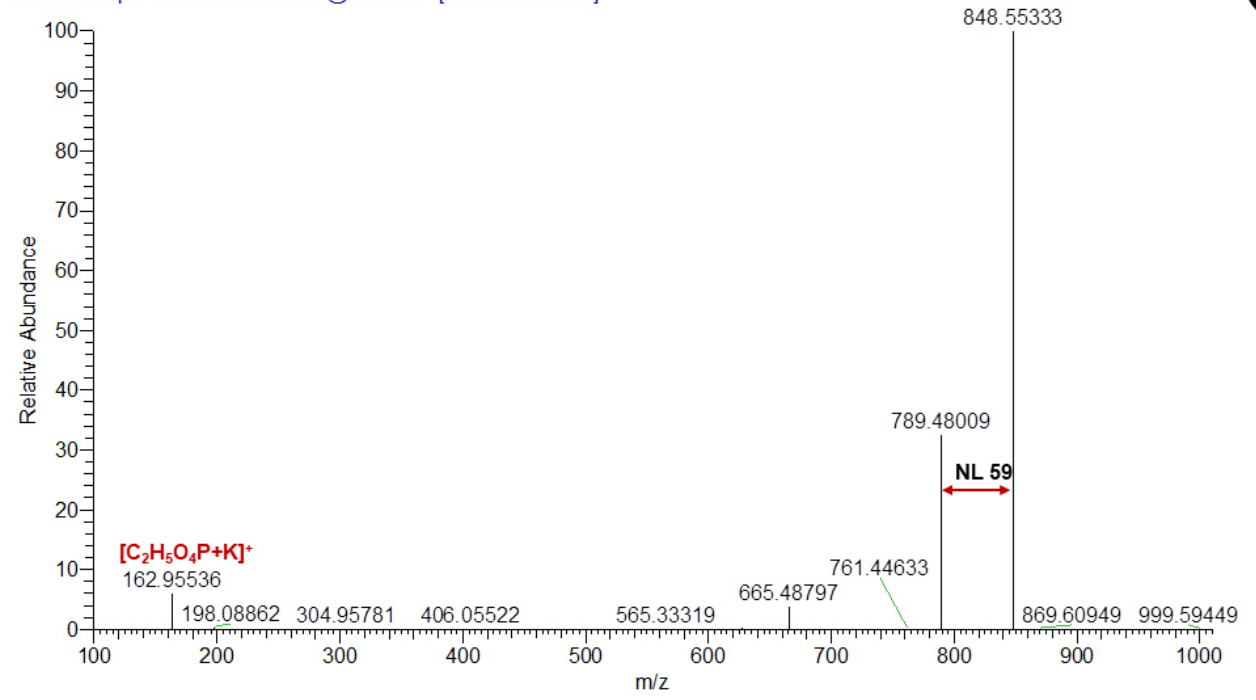


g)

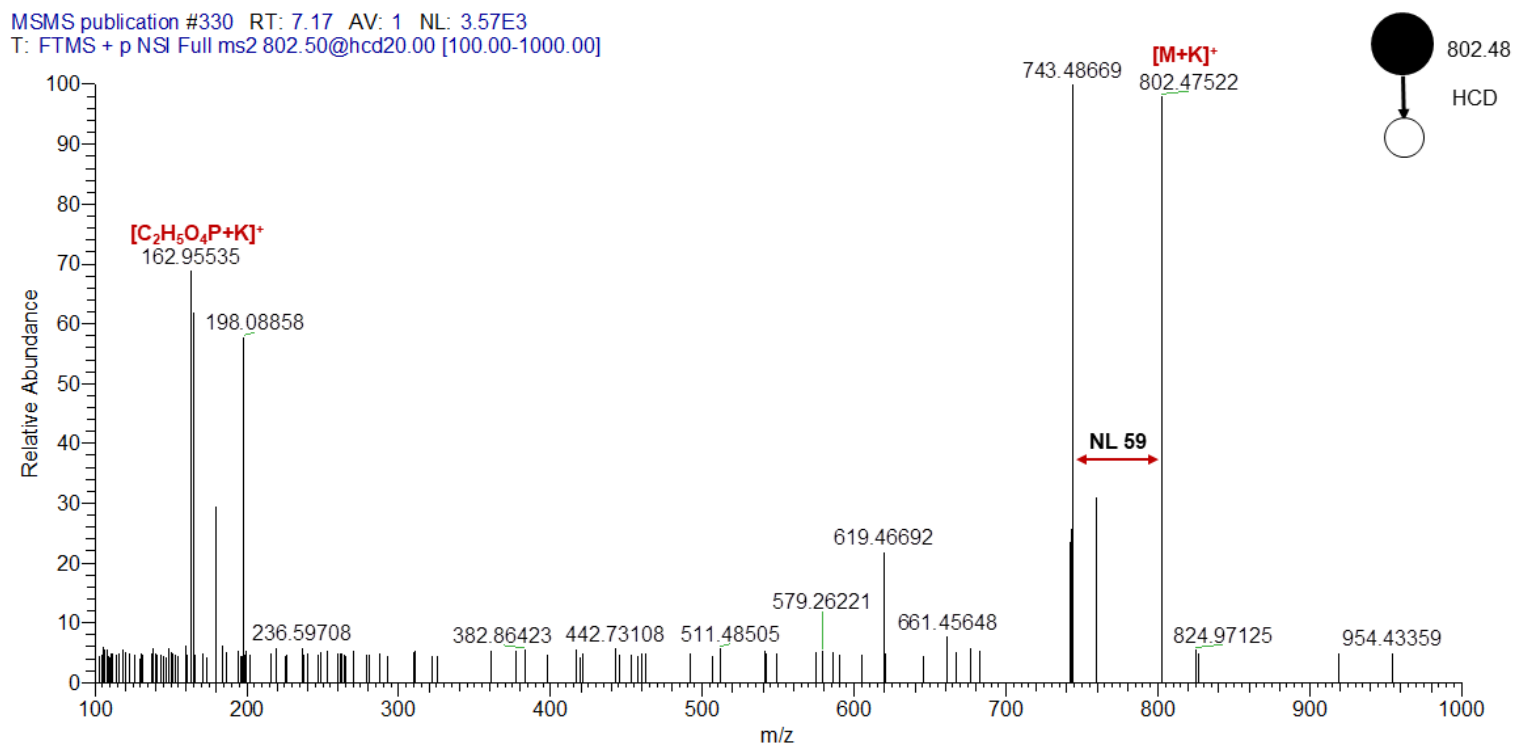

h)

$[\mathrm{PC}(38: 7)+\mathrm{K}]^{+}$

MSMS publication \#378-402 RT: $8.24-8.75$ AV: 25 NL: $2.84 \mathrm{E} 3$ T: FTMS + p NSI Full ms2 842.60@hcd20.00 [100.00-1000.00]
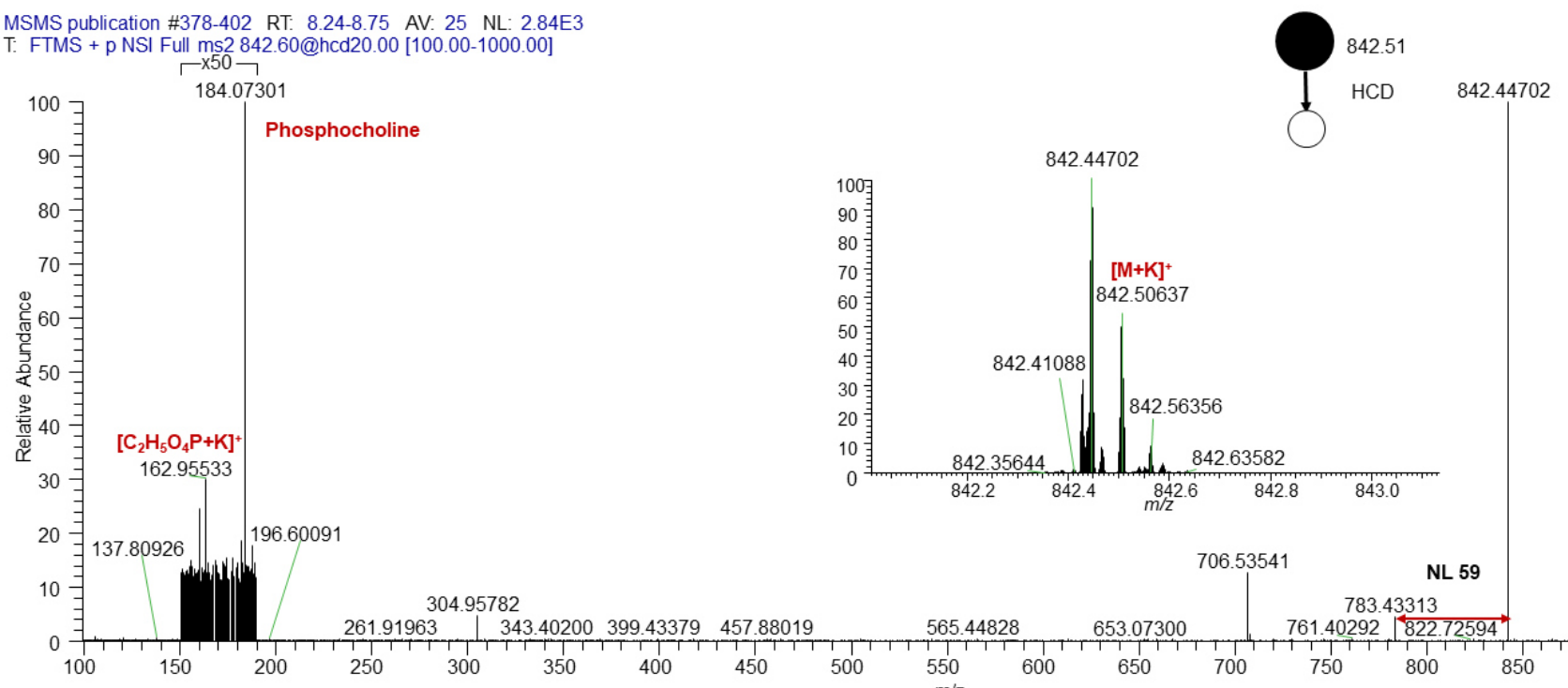

Supplementary Fig 4

On-tissue SMALDI MS/MS spectra for the selected mass peaks that are mentioned in the manuscript. Measurements were performed in positive ion mode to confirm the phospholipids' head group of each ion peak. a) MS/MS spectra of [LPC(16:0)+K] $]^{+}$ $\mathrm{m} / z 534.30$ see Fig. 3 and Tab. 1 ; b) MS/MS spectra of $[\mathrm{LPC}(18: 1)+\mathrm{K}]^{+} \mathrm{m} / z 560.31$ 
see Fig.1 and Tab. 1; c) MS/MS spectra of $[\mathrm{LPC}(18: 0)+\mathrm{K}]^{+} \mathrm{m} / z 562.33$ see Fig.1, Fig. 4 and Tab. 1; d) MS/MS spectra of $[\mathrm{PC}(36: 4)+\mathrm{K}]^{+} \mathrm{m} / z 820.53$ see Tab. 1; e) MS/MS spectra of $[\mathrm{PC}(38: 6)+\mathrm{K}]^{+} \mathrm{m} / z 844.53$ see Fig. 1 , Fig. 3 and Tab. 1 ; f) MS/MS spectra of $[\mathrm{PC}(38: 4)+\mathrm{K}]^{+} \quad \mathrm{m} / \mathrm{z} 848.56$ see Fig. 4 and Tab. $\left.1 ; \mathrm{g}\right) \mathrm{MS} / \mathrm{MS}$ spectra of $[\mathrm{PC}(38: 6)+\mathrm{K}]^{+} \quad m / z 802.48$ see Fig. 1 and Tab. 1 ; h) MS/MS spectra of $[\mathrm{PC}(38: 7)+\mathrm{K}]^{+}$ $m / z 842.51$ see Fig. 1 and Tab. $1 ; \mathrm{NL}=$ neutral loss 